\title{
Médiévales
}

Langues, Textes, Histoire

65 | automne 2013

Le couple dans le monde franc

\section{Le couple : une définition difficile, des réalités multiples}

What is a couple? Problematic definition, multiple realities

\section{Sylvie Joye et Emmanuelle Santinelli-Foltz}

\section{(2) OpenEdition}

1 Journals

\section{Édition électronique}

URL : https://journals.openedition.org/medievales/7073

DOI : 10.4000/medievales.7073

ISSN : 1777-5892

\section{Éditeur}

Presses universitaires de Vincennes

\section{Édition imprimée}

Date de publication : 1 décembre 2013

Pagination : $5-18$

ISBN : 978-2-84292-396-9

ISSN : 0751-2708

\section{Référence électronique}

Sylvie Joye et Emmanuelle Santinelli-Foltz, "Le couple : une définition difficile, des réalités multiples », Médiévales [En ligne], 65 | automne 2013, mis en ligne le 20 janvier 2014, consulté le 22 avril 2022.

URL : http://journals.openedition.org/medievales/7073; DOI : https://doi.org/10.4000/medievales. 7073 
Médiévales 65, automne 2013, p. 5-18

\author{
Sylvie JOYE et Emmanuelle SANTINELLI-FOLTZ
}

\title{
LE COUPLE : UNE DÉFINITION DIFFICILE, DES RÉALITÉS MULTIPLES
}

Si on a beaucoup parlé de la crise de la famille ${ }^{1}$, et si les polémiques sur le genre ont agité non seulement le monde universitaire mais aussi les médias en France il y a un ou deux ans ${ }^{2}$, c'est sur la définition du couple que se concentrent les débats depuis quelque temps, en particulier à l'occasion de la discussion autour du mariage des homosexuels en France et alors que la législation à ce sujet est aussi en pleine mutation dans une partie de l'Europe et de l'Amérique ${ }^{3}$. Si les enjeux de ces débats sont propres à nos sociétés contemporaines, certaines périodes passées ont déjà vu la réflexion sur le mariage, le couple et la famille prendre une grande importance et susciter difficultés et résistances. C'est en particulier le cas de l'Antiquité tardive et du haut Moyen Âge, où le couple est devenu un enjeu essentiel de la réflexion sociale et morale. Après avoir replacé

1. On peut citer le rapport sur la famille livré au Premier ministre français en 2005, à l'origine de la publication de M. Godet, É. Sullerot, La Famille : affaire privée et publique, Paris, 2007, qui, au sujet du couple, note en France la modification qui eut lieu entre 1999 et 2004 dans les critères du recensement (p.42). Un rapport précédent, par Irène Théry, mettait davantage la notion de couple en avant (du moins dans son titre, mais c'est bien la famille qui est désignée comme le sujet principal dans l'adresse à la ministre, p. 8): I. THÉRY, Couple, filiation et parenté aujourd'hui. Le droit face aux mutations de la famille et de la vie privée, Paris, 1998 (la réelle évaluation de ce qu'est un couple vient à la p. 143).

2. Sur le concept de genre et son utilisation par les historiens, et en particulier les médiévistes, voir dernièrement: D. LETT, «Les régimes de genre dans les sociétés occidentales de l'Antiquité au XVII ${ }^{\mathrm{e}}$ siècle », Annales. Histoire, Sciences Sociales, 67/3 (2012), p. 563-572.

3. V. Descoutures et al. éd., Mariages et homosexualités dans le monde. L'arrangement des normes familiales, Paris, 2008; B. Coulmont, «Mariage homosexuel, religion et État aux ÉtatsUnis », dans F. ROCHEFort éd., Le Pouvoir du genre. Laïcités et religions 1905-2005, Toulouse, 2007, p. 217-228. 
la question du couple dans les débats anthropologiques, l'analyse d'un extrait des Histoires de Richer permettra ici de préciser les problématiques pour le haut Moyen Âge, où la notion de couple et l'affirmation de sa place comme «structure portante» de la société connaissent des évolutions considérables.

\section{Questionnements anthropologiques}

\section{Quelle définition du couple?}

Les grandes figures des sciences humaines et de l'histoire, réagissant aux débats contemporains, se sont intéressées ces dernières années à la question du couple.Ainsi, Maurice Godelier, figure tutélaire de l'anthropologie de la parenté, a donné à l'occasion du récent débat sur le mariage un entretien au journal Le Monde le 17 novembre 2012 où il revient sur les liens entre mariage, famille et couple, s'interrogeant sur la place du couple dans la formation de l'individu. Paul Veyne avait déjà été l'un des premiers signataires d'un texte, paru dans le même journal en 1996 sous sa plume et celle de P. Bourdieu, J. Derrida, D. Eribon, M. Perrot et P. Vidal-Naquet, qui s'interrogeait sur la définition du couple: les premières discussions étaient alors engagées au sujet de la possibilité d'un contrat formant un couple pour les homosexuels aussi bien que pour les hétérosexuels. À l'époque, la jurisprudence disait clairement qu'un couple devait être composé d'un homme et d'une femme ${ }^{4}$.

Le couple est défini actuellement, et cette définition peut également valoir pour notre étude sur le haut Moyen Âge, par le partage entre deux personnes d'une sexualité reconnue socialement et accompagnée, mais ce n'est pas toujours le cas, de la cohabitation ${ }^{5}$. Le couple se trouve aussi à la conjonction de deux notions fondamentales, la famille et la parenté. Objet de toutes les attentions de la part des sociologues ${ }^{6}$, la notion de couple ne semble en revanche pas avoir tenu un rôle central dans les écrits anthropologiques français ${ }^{7}$ jusqu'à ces dernières

4. Article du Monde du $1^{\mathrm{er}}$ mars 1996.

5. I. ThÉRY, Couple..., p. 143: «C'est moins, est-il besoin de le dire, la réalité effective des relations sexuelles que leur licéité reconnue socialement qui distingue le couple d'un non-couple, et le concubinage des autres formes de cohabitation» (le terme «concubinage » étant pendant longtemps réservé en France aux couples de sexes différents, sous prétexte que celui-ci correspondait à une volonté de «vie maritale»).

6. Voir la récurrence du thème du couple dans le récent numéro des Cahiers français consacré à la famille: Comment va la famille?, Cahiers français, 371 (2012), p. 1-73.

7. La notion est absente de P. Bonte, M. Izard éd., Dictionnaire de l'ethnologie et de l'anthropologie, Paris, 1991 (la notice sur le mariage l'utilise sans la définir) comme du lexique du numéro de L'Homme dédié à la parenté (L'Homme, 154/155 [2000], p. 721-732). Dans C. LÉviSTrauss, Les Structures élémentaires de la parenté, Paris, 1967, le terme « couple» est essentiellement utilisé dans un sens technique bien précis (p. 148), et tout à fait différent des couples que nous étudions 
années ${ }^{8}$, peut-être parce que l'idée de l'échange des femmes par l'alliance et celle de filiation se trouvent finalement en deçà et au-delà du couple. On vit, il est vrai, en couple, mais cette notion n'est que très partiellement institutionnelle et la répartition des rôles, les relations et la constitution du couple ne se réduisent pas aux lois du mariage, pour ne citer que cet aspect. Les études de terrain, l'ethnologie et les réflexions de type plus culturaliste ont, de ce fait, centré davantage leur approche sur le couple. Le discours sur les liens entre public et privé, deux notions qu'on répugne à distinguer pour le haut Moyen Âge, et entre l'intime et le social, constitue un élément essentiel lorsque est abordée la notion de couple : celle-ci met l'accent sur la relation entre deux individus et pas seulement sur leur unité. Elle pointe en particulier la répartition des rôles et celle des attributs masculins et féminins entre homme et femme au sein du couple. Nous n'envisagerons en effet ici que des couples formés par un homme et une femme: les couples de même sexe apparaissent peu dans les sources et leur union ne semble pas bénéficier d'une réelle reconnaissance sociale ${ }^{9}$. La question d'une éventuelle existence de la polygynie, de son statut et des répercussions qu'elle peut avoir sur les relations entre les individus impliqués, est abordée par Régine Le Jan dans l'article qu'elle donne dans ce numéro de Médiévales. Si le mariage est l'objet d'un soin de plus en plus important au fil de la période, les couples qui existent en dehors du mariage ne peuvent être négligés ${ }^{10}$.

\section{Évolution du couple et transformations sociales et politiques}

Le discours et le personnel religieux jouent à la fin de l'Antiquité et durant le haut Moyen Âge un grand rôle, mais le christianisme n'est pas l'élément fondateur de l'idéologie du couple. Paul Veyne, dans son fameux article de

ici : reprenant une terminologie mise en place par Radcliffe-Brown, il évoque la paire (section des père et mère), le couple (section du père et de ses enfants, patrimoitié) et le cycle (section de la mère et de ses enfants, matrimoitié). Le terme «couple» dans son sens commun est utilisé au moment d'évoquer le «futur bonheur du couple» (p. 260) qui n'est pas menacé lorsque, dans le contexte socioculturel approprié, il ne semble pas excentrique.

8. L'index de M. Godelier, Métamorphoses de la parenté, Paris, 2004, comprend une large entrée consacrée au couple (p. 12-16, p. 577-580). Mais le terme n'apparaît pas dans le lexique final. Emmanuel Todd met en avant le thème du couple mais s'intéresse à lui comme «forme nucléaire pure» : E. TodD, L'Origine des systèmes familiaux, t. 1, L'Eurasie, Paris, 2011. Nous envisagerons donc ici bien le couple plus que la cellule conjugale, bien que la différence entre les deux notions soit ténue. Sur le point de vue du droit et de l'anthropologie juridique, voir: C. BonTEMs, «Le mariage: réflexions et inflexions», dans C. EBerhard, G. Vernicos éd., La Quête anthropologique du droit. Autour de la démarche d'É. Le Roy, Paris, 2006, p. $422-443$ (p. 430-431).

9. Malgré les tentatives pour les mettre en valeur de J. Boswell, Les Unions du même sexe dans l'Europe antique et médiévale, Paris, 1996. Voir M. KuEfLER éd., The Boswell Thesis, Chicago, 2005.

10. A. Esmyol, Geliebte oder Ehefrau? Konkubinen im frühen Mittelalter, Cologne, 2002. 
1978, a bien réfuté le lien classiquement établi entre moralisation de la famille et influence chrétienne. Il présente le nouveau statut du mariage, du couple et de l'idée d'amour conjugal comme le résultat d'une mutation intervenue durant les deux premiers siècles de notre ère et généralisée à l'époque des Sévère. La stabilité du couple et l'idée d'amour conjugal paraissent alors devenir la norme, et ce avant qu'ait pu s'exercer toute influence chrétienne, et sans que l'on puisse par ailleurs imputer ce changement à une prétendue morale stoïcienne, parfois conçue comme le terreau de cette mutation ${ }^{11}$. Mais le couple n'est qu'un épiphénomène de «l'histoire sexuelle romaine» (en fait l'histoire du discours sur la sexualité), qui constitue d'ailleurs, d'après Paul Veyne lui-même, l'objet réel de son article ${ }^{12}$. Pour lui, l'émergence du mariage, de la famille et de l'amour conjugal était avant tout une conséquence du changement d'attitude face à la sexualité, changement engagé durant les deux premiers siècles de notre ère qu'il liait à une mutation politique: soit le passage d'une «aristocratie concurrentielle», qui allait de pair avec un couple écrasé par la domination masculine et qui n'avait pas à se justifier par les sentiments, à une «aristocratie de service», qui «s'invente une morale conjugale et sexuelle, afin que la discipline lui vienne de nouveau de l'extérieur». Cette fois encore, cependant, il faut prendre en compte les effets qu'entraîne le recours aux sources et les propositions de Paul Veyne ont pu être contestées par des auteurs qui y sont retournés ${ }^{13}$. Il n'en demeure pas moins qu'il faut sans doute lier l'attention nouvelle portée au couple à des transformations sociales et politiques, et qu'il faut surtout se garder de la rattacher uniquement à la christianisation de l'Occident.

Un lien aussi direct entre évolution du couple et évolution sociale et politique n'a pas été mis en lumière par les médiévistes, même s'il faut noter les

11. P. Veyne, «La famille et l'amour sous le Haut-Empire romain», dans ID., La Société romaine, Paris, 2001, p. 90-96 [1 ${ }^{\text {re }}$ éd. 1978].

12. Ibid., p. 88. Pour le Moyen Âge, on peut rappeler un article centré sur les notions de couple et d'amour, dans une tout autre perspective: M. Charageat, «Couples et amour en Aragon ( $\mathrm{XV}^{\mathrm{e}}$ $\mathrm{XvI}^{\mathrm{e}}$ s.)», Clio, 34 (2011), p. 41-60.

13. Critique de l'article, notamment sur les liens des hypothèses de P. Veyne avec les vieilles théories de la décadence romaine: M. BÉNAMOU, «Pratique matrimoniale et représentation philosophique : le crépuscule des stratégies? », dans J. ANDREAu, H. BRuHns éd., Parenté et stratégies familiales dans l'Antiquité romaine, Rome, 1990, p. 129-130. P. Veyne se réclame lui-même de Max Weber, et André Burguière ajoute à cette référence celle de Norbert Élias qui liait la construction de l'État monarchique et l'avènement de la société de cour: A. BuRGuiÈRE, «De la Rome républicaine à la Chrétienté post-tridentine. Les raisons du mariage dans la très longue durée », dans J. ANDREAU, H. Bruhns éd., Parenté..., p. 290. Mise au point récente par S. Dixon, «The Sentimental Ideal of the Roman Family», dans B. Rawson éd., Marriage, Divorce and Children in Ancient Rome, Oxford,

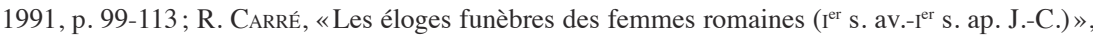
dans D. Jonckers, R. CARré, M.-C. Dupré éd., Femmes plurielles. Les représentations des femmes. Discours, normes et conduites, Paris, 1999, p. 121-137. 
recherches récentes que Tiziana Lazzari a consacrées à des modèles de couples dont la condamnation, par les lois et les conciles, semble impliquer le refus de certains modes de fonctionnement économiques et communautaires dans $1^{\prime}$ Espagne wisigothique et l'Italie lombarde ${ }^{14}$. La valorisation du couple à la période carolingienne a cependant été bien soulignée par Pierre Toubert ${ }^{15}$ et Régine Le Jan ${ }^{16}$. Le couple est un sujet qui revêt alors une grande importance pour les autorités (diverses), en ce qu'il est conçu à la fois comme l'atome essentiel de la société et le modèle des bonnes relations entre individus dans tous les domaines: image alors bien mise en avant, mais qui n'est sans doute pas caractéristique uniquement de l'époque carolingienne, où les sources théoriques sur le sujet se multiplient. En ce domaine, il faut surtout faire la part des constructions idéologiques et des réalités, et s'interroger sur les relations existant entre les unes et les autres.

Si le modèle du couple peut être transféré du haut de la société vers le bas (ou inversement des strates les plus modestes aux plus élevées), il faut se garder de trop généraliser nos observations à tous les niveaux de la société ou à tous les types d'organisation ou d'habitat. Même dans un milieu précis, les données et les évolutions sont complexes : ainsi Kate Cooper a bien montré le paradoxe, du point de vue des femmes, de ce que signifia la promotion du couple et de l'idéal conjugal au IV siècle, notamment parce qu'il fut contemporain d'une très grande valorisation de l'ascétisme chez les dames de l'aristocratie romaine. L'influence morale de la femme dans le couple et la famille devient du même coup, cependant, un thème majeur à partir de cette époque ${ }^{17}$.

Même si les études réunies dans ce volume traitent surtout des dames de l'aristocratie et de la théorie du couple idéal, les auteurs ont cherché à y faire apparaître, autant que possible, les traces des relations, bonnes ou mauvaises, qu'entretenaient les individus vivant en couple, et pas seulement leurs rôles tout constitués. Une grande place est faite au partage (des biens certes, mais aussi des sentiments ou du travail) pour discerner un couple qui vit au rythme de cycles et de reconfigurations.

14. T. LAZZARI, «"Secondo natura": l'età giusta per il matrimonio nelle leggi dell'Europa altomedievale», dans M. DAvidE éd., La condizione giuridica delle donne nel medioevo, Trieste, 2012, p. 27-51.

15. P. TOUBERT, «La théorie du mariage chez les moralistes carolingiens», dans Matrimonio nella società altomedievale, Settimane di Studio del centro italiano di studi sull'alto medioevo, Spolète, 1976, p. 233-281.

16. R. LE J JN, Famille et pouvoir dans le monde franc (VII $-X^{e}$ s.). Essai d'anthropologie sociale, Paris, 1995, p. 333-379 (chap. x, «Autour du couple conjugal»).

17. K. COOPER, The Fall of the Roman Household, Cambridge, 2007; EAD., «Poteri invisibili: la matrona cristiana tra obsequium e autorità legittima all'epoca di sant'Agostino », dans C. LA RoccA éd., Agire da donna. Modelli e pratiche di rappresentazione (sec. VI-X), Turnhout, 2007, p. 11-21. 


\section{Le mariage raté de Louis $V$ et Adélaïde, d'après Richer}

Si certains questionnements sont universels (ce qui ne signifie pas qu'ils se posent de la même manière à toutes les époques), d'autres sont propres au haut Moyen Âge, à sa société et aux sources qui l'éclairent. L'analyse du récit que fait Richer, à la fin du $x^{\mathrm{e}}$ siècle, du mariage raté du jeune roi Louis $\mathrm{V}$ au début des années 980 , permettra de soulever les problèmes de la documentation, de faire le point sur les données fournies par les travaux récents sur les premiers siècles médiévaux, les femmes ou le genre, et de proposer des questionnements sur la thématique du couple entre les $\mathrm{VI}^{\mathrm{e}}$ et $\mathrm{XII}^{\mathrm{e}}$ siècles. Selon le moine de SaintRemi de Reims :

Comme le roi [Lothaire] voulait que son fils Louis lui succédât sur le trône [...] Louis fut proclamé roi (c. 91).

$[\ldots]$ De rusés personnages [...] allèrent trouver la reine Emma [...]. Ils lui dirent que ce serait une excellente chose que de faire épouser, au roi Louis, Adélaïde, épouse (uxor) de Raimond, duc des Goths, mort récemment. Du fait de ce mariage, [...] sa capacité d'exercer le pouvoir en serait accrue [...] il lui serait possible de soumettre à sa domination tout à la fois l'ensemble de l'Aquitaine et la Gothie, après avoir transféré les places les plus fortifiées du droit de sa nouvelle épouse (ex jure ductae uxoris) au sien; de plus [...] le père et le fils, installés, l'un en deçà et l'autre au-delà, pourraient sans relâche menacer le duc [Hugues Capet] et le reste de leurs ennemis ainsi encerclés au milieu (c. 92).

[...] Le projet qui avait été suggéré au roi, fut adopté en présence du comte Geoffroi [d'Anjou]. [...] Les deux rois partirent pour l'Aquitaine [...] (c. 93). Adélaïde les reçut avec grand apparat. [...] Le roi Louis l'épousa (eam sibi uxorem copulavit) et l'éleva à la royauté en la faisant couronner par les évêques. [...] L'amour conjugal (amor conjugalis) était chez eux presque nul. Comme lui était tout juste pubère (adhuc pubesceret) et elle déjà une vieille femme (anus foret), ils ne s'entendaient pas du fait de leurs caractères contraires (contrariis moribus dissentiebant). Ils refusaient de faire lit commun (cubiculum commune) et pour se reposer, ils rejoignaient des toits différents (divertiis hospitiis potiebant). Lorsqu'ils avaient à s'entretenir, ils le faisaient dans un lieu en plein air. Leur conversation se bornait à quelques paroles très brèves. Ceci dura entre eux près de deux ans; puis leur désaccord devint tel qu'un divorce (divortium) ne tarda pas à s'en suivre (c. 94).

$[\ldots]$ La reine $[\ldots]$ se rendit auprès de Guillaume d'Arles et l'épousa. Son divorce devint ainsi un adultère public (adulterium publicum) (c. 95) ${ }^{18}$.

18. Richer, Historiarum libri IV, III, 91-95 (éd. et trad. R. LATOuche, Histoire de France [888995], t. II, Paris, 1937, p. 114-121). La traduction de plusieurs passages est personnelle. 


\section{Repérer les couples dans les sources}

Le récit évoque deux couples: d'une part, celui formé par le roi Lothaire (954-986) et la reine Emma; d'autre part, celui constitué par leur fils Louis V et Adélaïde ${ }^{19}$. Il s'agit de couples, au sens de l'union d'un homme et d'une femme qui présente une certaine stabilité, notamment du fait de la conclusion d'un mariage, mais que l'auteur ne présente pas agissant conjointement ou se comportant véritablement comme maris et femmes. En ce qui concerne le premier, Richer ne parle que du roi et de la reine, sans préciser qu'ils sont mariés - ni avoir évoqué dans les chapitres précédents leur mariage ou les avoir montrés ensemble: il évoque tantôt l'un, tantôt l'autre, même si l'on suppose que «les rusés personnages» s'adressent à la reine avant de se tourner vers le roi, dans l'espoir qu'elle intervienne pour convaincre ce dernier, ce qui implique des discussions entre époux. Si l'auteur ne mentionne pas la reine aux côtés du roi lorsque le projet de mariage est adopté, puis lorsque Lothaire conduit son fils pour le marier en Aquitaine, cela ne signifie pas qu'elle n'était pas présente, compte tenu de son rôle par ailleurs attesté ${ }^{20}$, mais Richer se focalise sur les hommes. Quant au second couple, celui formé par Louis V et Adélaïde, on sait qu'il en est un, puisque Richer évoque le mariage, mais celui-ci montre ensuite que les deux époux ne se comportent pas comme tel.

Les extraits posent donc la question du repérage des couples dans les sources. Ceux-ci sont identifiés par déduction: comme ici, parce que l'auteur évoque un mariage ou parce qu'il mentionne deux individus de sexes différents de même statut (royal), dont l'union est confirmée par un autre passage ou une autre source; plus souvent, parce que la documentation - narrative, mais aussi diplomatique, nécrologique, voire administrative avec les polyptyques - met en scène des hommes et/avec leur femme (uxor, conjux), voire plus rarement des femmes et/avec leur mari (maritus, coniux), ou encore parce qu'elle évoque le père et la mère d'un individu. Le pluriel (conjuges) n'est que rarement utilisé pour désigner les époux. Quant au terme parentes, $\mathrm{s}$ 'il désigne parfois le père et la mère envisagés ensemble, il renvoie plus souvent aux membres de la parenté et, même dans le premier sens, il désigne le couple, non dans le rapport qui existe entre

19. Sur ces deux couples, voir notamment F. Lot, Les Derniers Carolingiens. Lothaire, Louis V, Charles de Lorraine (954-991), Paris, 1891; R. LE JAN, «D'une cour à l'autre: les voyages des reines de Francie au xe siècle», dans EAD., Femmes, pouvoir et société, Paris, 2001, p. 38-52; T. STASSER, «Adélaïde d'Anjou, sa famille, ses unions, sa descendance. État de la question », Le Moyen Âge, 103 (1997), p. 9-52; E. MAGnANI, «Les femmes et l'exercice du pouvoir comtal dans le Midi. Autour d'Adélaïde Blanche d'Anjou, comtesse de Provence (1026)», dans A. NAYT-Dubois, E. SANTinelliFoltz éd., Femmes et pouvoir des femmes dans l'Occident médiéval et moderne, Valenciennes, 2009, p. 273-280.

20. R. LE J J , «D'une cour à l'autre...». 
conjoints, mais dans sa relation aux enfants. Quelques moralistes carolingiens considèrent certes que les époux forment un consortium $^{21}$, ce qui montre qu'ils envisagent l'homme et la femme mariés comme une entité propre. Mais, en dehors de cet usage, il faut attendre le milieu du XII siècle pour que soit utilisé le terme spécifique de «couple» (copula; cuple) pour désigner l'union d'un homme et d'une femme ${ }^{22}$. Si le terme de couple n'existe pas au haut Moyen Âge, en revanche, les sources mentionnent parfois l'adjectif «conjugal» (conjugalis), à l'image de «l'amour conjugal» évoqué par Richer.

L'usage de l'adjectif, ainsi que la mention de certaines pratiques, montrent que l'absence d'un substantif spécifique n'exclut pas la réalité du couple ni une certaine conscience de son existence. Le manque de vocabulaire distinctif entraîne néanmoins des difficultés de repérage (outre les périphrases qui excluent le recours aux index, il faut parfois croiser plusieurs extraits, voire plusieurs sources, pour identifier les couples). Il invite, en outre, à s'interroger sur la perception du couple: si certains moralistes le considèrent comme une communauté de vie et d'affection, qu'en est-il du reste de la population, tant des autres élites pensantes que des hommes et femmes mariés? Ce consortium proposé en modèle aux époux est-il un idéal pour tous? Et trouve-t-il des échos dans la société? Ces questions méritent d'autant plus d'être posées que, si certaines sources évoquent côte à côte des époux ou des parents qui forment, à nos yeux du moins, des couples, elles mentionnent beaucoup plus souvent des hommes - et plus rarement des femmes - agissant seuls, du moins sans conjoint, ce qui ne signifie pas qu'il n'y en ait pas, ni même que celui-ci soit absent.

\section{Couples et groupes familiaux}

Dans les extraits sélectionnés, il est d'abord question du mariage de Louis V et d'Adélaïde, acte créateur du couple. Richer met alors en lumière deux idées: d'une part, le mariage de Louis est lié à l'association de celui-ci à la royauté (879), consécutive à son passage à l'âge adulte; d'autre part, il s'inscrit dans les stratégies familiales. Élevé à la royauté par son père alors qu'il est «tout juste pubère ${ }^{23}$, Louis V est établi : il reçoit donc à ce titre un royaume (l'Aquitaine) et une épouse (Adélaïde). Le choix de celle-ci ne revient pas à Louis V, mais à ses parents, et n'est pas guidé par les qualités particulières d'Adélaïde, même si certaines ont pu entrer en ligne de compte, mais par les stratégies familiales. Le projet est négocié, non par les intéressés, mais, conformément

21. P. TOUBert, «La théorie du mariage... ».

22. A. WALCh, Histoire du couple en France de la Renaissance à nos jours, Rennes, 2003, p. 9; A. Rey éd., Dictionnaire historique de la langue française, Paris, rééd. 1993, p. 513.

23. Louis V, né vers 967, a une douzaine d'années en 979. 
aux pratiques d'alors, par le roi Lothaire, père du futur marié, et par le comte Geoffroi Grisegonelle d'Anjou, frère d'Adélaïde, qui exerce l'autorité sur le groupe familial de la promise - et notamment les enfants, les filles non mariées et les femmes devenues veuves - depuis que son père Foulques II le Bon (960) est mort. Richer semble en faire une initiative des Angevins, si l'on considère que les rusés personnages qui vont trouver la reine Emma sont des fidèles du comte d'Anjou: le mariage de sa sœur avec le roi doit lui permettre de renforcer son alliance avec les Carolingiens et de s'élever dans la hiérarchie princière, ce qui le favorise sur l'échiquier des forces aux dépens de ses voisins et rivaux, Hugues Capet et Guillaume IV d'Aquitaine. Richer insiste cependant sur les avantages en termes de pouvoir que la royauté peut retirer de ce mariage.

La formation du couple - par le biais du mariage -, avec son rôle dans les stratégies familiales, mais aussi ses étapes et les transferts de biens, voire de pouvoir, auxquels elle donne lieu, est aujourd'hui mieux connue ${ }^{24}$, même si l'on mesure mal la conscience qu'en avaient les individus: estimait-on que l'union légitime d'un homme et d'une femme aboutissait à la construction d'une entité se pensant et agissant solidairement? Il n'en demeure pas moins que Louis V est conduit à sa femme et reste en Aquitaine : même si le couple se trouve inséré dans des groupements plus larges dont il sert les intérêts, il vit au quotidien de manière autonome. Régine Le Jan a montré en outre que les interdits de parenté, durcis à partir de l'époque carolingienne par les autorités religieuses, avaient contribué, en luttant «contre les pratiques qui sous-tendaient les solidarités familiales», à promouvoir le couple conjugal ${ }^{25}$. Pour autant, il reste à préciser comment s'est traduite concrètement la promotion du couple.

\section{Quel couple?}

Richer précise qu'Adélaïde, donnée en mariage à Louis, est veuve de Raimond de Toulouse - épousé après la mort d'un premier mari, Étienne de Brioude. En outre, après avoir divorcé de Louis V, Adélaïde se remarie avec Guillaume de Provence (qui était veuf) ${ }^{26}$. La forte mortalité - masculine (activités guerrières, écart d'âge au mariage), mais aussi féminine (procréation), même si celle-ci est un peu moindre ${ }^{27}$ - et, plus rarement, les séparations conduisent à

24. R. LE JAN, Famille et pouvoir..., p. 263-327; M. AurelL éd., Les Stratégies matrimoniales (IX $X^{e}$-XIII S.), Actes du colloque de Poitiers (25-26 mai 2012), à paraître.

25. R. LE JAN, Famille et pouvoir..., p. 315.

26. Sur les quatre maris successifs d'Adélaïde, voir T. STASSER, «Adélaïde...», p. 14-21; E. Magnani, «Les femmes...», p. 274-276.

27. E. SANTINelli, Des femmes éplorées? Les veuves dans la société aristocratique du haut Moyen Âge, Lille, 2003, p. 47-58: la probabilité de connaître le veuvage semble deux fois plus élevée pour une femme qu'un homme. 
multiplier les unions successives et, par conséquent, à faire partie de plusieurs couples: quatre pour Adélaïde, ce qui n'est pas exceptionnel, même si les remariages d'un individu - pratique des plus courantes, pour les hommes comme pour les femmes ${ }^{28}$ - sont généralement moins nombreux. Cela pose la question de savoir comment sont appréhendés ces différents couples auxquels un même individu peut avoir appartenu et comment le souvenir de ces couples successifs est entretenu. Il n'est pas inintéressant de noter que Richer présente Adélaïde comme «épouse» (uxor) d'un mari récemment décédé, et non comme veuve (vidua ou relicta) de celui-ci: peut-être du fait des droits auxquels celle-ci peut prétendre comme (ex-)épouse du défunt ${ }^{29}$. En revanche, Richer occulte le premier mariage d'Adélaïde, et donc son premier couple - connu par d'autres sources -, parce que cela n'entre pas dans son propos.

Plus généralement, les sources montrent que les contemporains sont attentifs au souvenir des différents couples auquel un même individu a pu participer, du moins en certaines occasions : c'est notamment le cas lorsqu'il est question des enfants et de la memoria. Les enfants sont, en effet, régulièrement présentés comme fils ou filles d'un tel et d'une telle, donc comme enfants d'un duo de parents, même si l'objectif n'est pas tant de conserver le souvenir du couple que des droits, matériels et immatériels, auxquels l'individu peut prétendre du fait de sa mère et de son père. Lorsqu'un homme ou une femme a eu des enfants de plusieurs partenaires successifs, il arrive aussi régulièrement que soit précisé de quelle union ceux-ci sont nés. Le souvenir du couple, voire des différents couples, dont un individu a pu faire partie est aussi entretenu par le biais de la memoria (mémoire liturgique, par le biais de prières dites en faveur de l'âme des défunts), dont la pratique se développe à l'époque carolingienne. Les chartes enregistrent ainsi des donations réalisées, à charge pour les religieux bénéficiaires de prier pour les père et mère du donateur, voire pour un conjoint décédé. Malgré le caractère temporaire des couples, lié aux aléas de la vie, ces derniers peuvent garder par ce moyen une certaine pérennité au-delà de leur dissolution: il convient néanmoins de préciser la fréquence de cette pratique, ses formes et les évolutions observables.

La dernière précision donnée par Richer dans les extraits cités, concernant le remariage d'Adélaïde, pose en outre la question de la définition du couple et des différentes façons de l'envisager: à une époque où l'Église cherche à imposer plus fermement l'indissolubilité du mariage ${ }^{30}$, l'union d'Adélaïde qui suit son divorce avec Louis V est qualifiée «d'adultère public» par l'auteur, qui

28. Ibid., p. 243.

29. Ibid., p. 243-252.

30. E. SANTinelli éd., Répudiation, séparation, divorce dans l'Occident médiéval, Valenciennes, 2007, notamment «Introduction», p. 9-31 (p. 14-19). 
considère comme plusieurs de ses contemporains ecclésiastiques qu'Adélaïde ne peut se remarier tant que Louis V est vivant. Cette nouvelle union n'en est pas moins considérée comme légitime par la majorité des élites locales, mais aussi du royaume : les critiques dont fait l'objet Constance, née de l'union d'Adélaïde et de Guillaume de Provence et devenue reine après avoir épousé le roi Robert le Pieux, ne font aucunement allusion à une naissance illégitime, y compris chez les auteurs qui, comme Raoul Glaber, l'apprécient peu et n'auraient pas manqué de le faire si l'union de ses parents avait été reconnue nulle. Dans tous les cas, il y a formation d'un nouveau couple, mais sa nature n'est pas envisagée de la même manière par les contemporains selon qu'ils considèrent l'union comme légitime ou non. Les sources, émanant essentiellement des milieux ecclésiastiques, évoquent davantage, lorsqu'elles mentionnent des couples, ceux issus d'un mariage légitime que ceux qui ne le sont pas, ce qui ne signifie pas qu'il faille les occulter. Outre leur fréquence, il convient de préciser leur particularité - notamment en termes de durée et de fonctionnement - par rapport aux couples légitimes.

\section{Couple et pouvoir}

Richer présente ensuite l'association d'Adélaïde au pouvoir royal comme une conséquence du mariage: le mariage avec un roi entraîne l'élévation à la royauté symbolisée par le couronnement. L'époque carolingienne est aussi marquée, en effet, comme l'a montré Régine le Jan, par une association plus étroite de l'épouse à l'exercice du pouvoir, d'abord à l'échelon royal, avec une intensification au $\mathrm{x}^{\mathrm{e}}$ siècle, avant de se diffuser dans les milieux princiers et le reste de l'aristocratie: l'organisation des groupements de parenté en structures plus verticales a même, en associant plus étroitement l'épouse à son mari, renforcé la conjugalité ${ }^{31}$. La manière dont cette association s'est traduite est de mieux en mieux connue ${ }^{32}$, mais les analyses portent davantage sur les conséquences qui en résultent pour l'épouse (notamment ses nouvelles fonctions) que sur ce qui pourrait conduire à promouvoir le couple en tant qu'unité solidaire de décision et d'action (par exemple, les actes réalisés conjointement et les symboles de pouvoir communs), ce qui reste donc à préciser.

\section{Couple et vie conjugale}

Enfin, l'essentiel du chapitre 94 est consacré à la liste de tous les dysfonctionnements qui semblent caractériser la vie du couple formé par Louis $\mathrm{V}$

31. R. LE JAN, Famille et pouvoir..., p. 356-365.

32. Ibid.; T. Evergates éd., Aristocratic Women in Medieval France, Philadelphie, 1999. 
et Adélaïde et aboutissent à la rupture. Cette succession de remarques, qu'elles correspondent à la réalité ou non, permet d'avoir quelques informations sur l'idée que Richer, et probablement une partie au moins de ses contemporains, se fait du couple. L'auteur commence par souligner l'absence d' « amour conjugal », ce qui est assez surprenant car, pour les moralistes carolingiens, s'il devait y avoir affection (dilectio, caritas) entre les époux, il fallait éviter l'amour (amor), source de bien des maux ${ }^{33}$. Pour Richer, le couple implique qu'il y ait amour entre époux. Si mari et femme, du moins lorsqu'il y a mariage, se trouvent unis sans l'avoir choisi, cela n'exclut pas - sans que cela soit toutefois automatique - la naissance d'une affection conjugale, voire d'un véritable amour, entre les époux, amenés à partager la vie quotidienne avec ses joies et ses difficultés ${ }^{34}$. L'entente n'est cependant pas garantie, comme le récent colloque sur la parenté déchirée $e^{35}$ en a notamment donné une belle collection d'exemples, auxquels s'ajoute donc le cas de Louis V et Adélaïde. Richer donne ensuite une explication à l'absence d'amour conjugal: les dissentiments nés de caractères différents liés à un écart d'âge important, Adélaïde ayant, avec ses 30 ans environ, plus du double de l'âge de Louis ${ }^{36}$. Il est possible qu'un décalage dans ce sens ait davantage posé de problèmes que dans le cas, beaucoup plus fréquent, d'un époux nettement plus âgé que sa femme. Il est cependant peu probable que cela soit la véritable raison des conflits conjugaux - si tant est que ceux-ci aient véritablement existé.

Richer précise ensuite que les époux ne faisaient pas lit commun, ce qu'il faut comprendre - du fait de la suite de la phrase - par «ils n'avaient pas de relations sexuelles». L'absence d'amour conjugal n'exclut pas la copulation au sein du couple. La remarque peut surprendre sous la plume d'un clerc, à une époque où les milieux ecclésiastiques, tout en reconnaissant que le mariage implique union charnelle, en recommandent un usage modéré, en vue de la procréation. Richer apparaît donc conscient des réalités de la vie conjugale et finalement plus proche de celles-ci que du discours théorique des clercs. En outre, même pour se reposer (c'est-à-dire en dehors des relations sexuelles), Louis V et Adélaïde vivent dans des demeures séparées. Incontestablement, le couple implique la communauté de vie, dans une même maison - dont les sources, écrites comme archéologiques, témoignent qu'elle était conçue pour un seul couple -,

33. P. TOUBerT, «L'institution du mariage chrétien, de l'Antiquité tardive à l'an Mil», dans Settimane di Studio del centro italiano di studi sull'alto medioevo, Spolète, 1998, p. 503-553 (p. 524526).

34. I. RÉAL, Vies de saints, vie de famille. Représentation et système de la parenté dans le royaume mérovingien (481-751), d'après les sources hagiographiques, Turnhout, 2001, p. 348-368; D. LetT, Famille et parenté dans l'Occident médiéval, $V^{e}-X V^{e}$ siècle, Paris, 2000, p. 179-181.

35. M. Aurell éd., La Parenté déchirée: les luttes intrafamiliales au Moyen Âge, Turnhout, 2010.

36. T. STASSER, «Adélaïde...», p. 20. 
ce qui n'exclut pas des séparations temporaires liées aux impératifs politiques, militaires, économiques ou religieux ${ }^{37}$. Divers indices laissent supposer que durant tout le haut Moyen Âge, de manière générale, les époux partageaient la même chambre et le même lit. Enfin, Richer constate que Louis $\mathrm{V}$ et Adélaïde n'échangent que de brèves paroles en guise de conversation. La vie de couple implique aussi des échanges verbaux, les sources soulignant parfois le rôle de conseillère joué par l'épouse auprès de son mari ou son association à certaines décisions, ce que suggère d'ailleurs Richer pour la reine Emma.

\section{Données originales, informations partielles}

Si tous ces constats faits par Richer donnent un éclairage original, pour le $\mathrm{X}^{\mathrm{e}}$ siècle, sur le couple - celui de Louis V et Adélaïde, celui de Lothaire et Emma, mais aussi le couple en général -, il reste à le comparer avec d'autres données fournies pour d'autres périodes et d'autres milieux sociaux, et à le compléter pour saisir plus précisément ce qu'est un couple au haut Moyen Âge. Considèret-on qu'un homme et une femme unis l'un à l'autre vivent et agissent ensemble - ce qui ne signifie pas forcément de la même manière? Sont-ils envisagés et se pensent-ils - en tant que communauté solidaire - ce qui ne signifie pas égalitaire ? Comment les relations au sein du couple sont-elles pensées et vécues au quotidien? Richer, comme la plupart de ses contemporains, enregistre d'abord ce qui concerne les élites, ici royales, mais les indications fournies par d'autres sources, comme les polyptyques, les formulaires ou la littérature hagiographique, témoignent que le couple est aussi une réalité dans le reste de la société, même si l'on en perçoit encore plus mal les implications concrètes. Si le sommet de la hiérarchie sociale est mieux éclairé, il n'en demeure pas moins que les informations données par Richer dans les extraits précédemment analysés sont peu communes et qu'elles ne sont fournies que parce qu'il y a dysfonctionnement (et donc dignes d'être notées). D'une manière générale, la documentation est avare de données sur le sujet. Les remarques précédentes ne doivent pas occulter le fait, comme on l'a déjà évoqué, que la documentation éclaire d'abord et surtout les hommes, et ne mentionne souvent leurs épouses que pour l'alliance qu'elles permettent avec leur famille et les enfants qui leur naissent: faut-il en déduire que cette image floue s'explique parce que le couple est une réalité évidente pour tous et donc inutile à évoquer? Ou bien parce qu'on n'a pas véritablement conscience de son existence?

La documentation n'est pas certes aussi riche qu'on le souhaiterait. Elle n'en fournit pas moins des informations qui deviennent plus abondantes aux $\mathrm{X}^{\mathrm{e}}-\mathrm{XI}^{\mathrm{e}}$ siècles, même si elles sont clairsemées et que l'intimité du couple reste

37. I. RÉAL, Vies de saints..., p. 305-320; R. LE JAN, Famille et pouvoir..., p. 334-339. 
difficilement pénétrable. L'objectif de ce volume consiste donc à en glaner quelques-unes de manière à saisir le couple du haut Moyen Âge, dans la réalité quotidienne et dans la perception que l'on en avait, ainsi que les évolutions qui affectent l'une et l'autre. Les analyses seront envisagées à différents niveaux : d'abord, celui des époux qui le forment, puis celui des groupes familiaux dans lesquels il s'insère - Sippen qui se transforment progressivement aux $\mathrm{X}^{\mathrm{e}}-\mathrm{XI}^{\mathrm{e}}$ siècles en lignages -, enfin celui des législateurs et des moralistes, voire des artistes, dont la vision est plus globale. Si les transformations survenues à l'époque carolingienne, qui conduisent à renforcer la place du couple au sein des groupements de parenté, ont été soulignées à plusieurs reprises, il reste à préciser les évolutions entre le $\mathrm{VI}^{\mathrm{e}}$ et le $\mathrm{XII}^{\mathrm{e}}$ siècle, tant dans les comportements au sein du couple que dans la place qui lui est faite par les groupes familiaux et dans les manières de l'envisager plus ou moins consciemment dans son essence, mais aussi de préciser sa place et son rôle dans la société. De manière à ne pas éparpiller les approches, le cadre spatial a été volontairement restreint à l'espace franc, ce qui n'empêchera pas de souligner d'éventuelles différences régionales. Les articles qui suivent cherchent à diversifier les perspectives, tant sur le plan des thématiques (précédemment soulevées) et des périodes (mérovingienne, carolingienne, temps des principautés) que des milieux sociaux (élites, mais aussi monde paysan) et des sources (narratives et législatives, mais aussi traités, actes de la pratique et enluminures). Nous n'avons nullement la prétention de traiter la question de manière exhaustive, mais seulement de proposer quelques sondages qui permettent de défricher un sujet qui mériterait une synthèse générale.

Sylvie Joye - Université de Reims, Département d'Histoire, 57 rue PierreTaittinger, 51096 Reims Cedex

Emmanuelle Santinelui-Foltz - Université de Valenciennes, Département d'Histoire, Le Mont-Houy, BP 311, 59304 Valenciennes Cedex 